\title{
Biological activities specified by antibiotic resistance plasmids*
}

\author{
Kenneth N. Timmis, Manuel I. Gonzalez-Carrero, Tsutomu Sekizaki \\ and Fernando Rojo \\ Department of Medical Biochemistry, University of Geneva, Switzerland
}

\begin{abstract}
Bacteria can display resistance to a wide spectrum of noxious agents and environmental conditions, and these properties are often mediated by genes located on extrachromosomal DNA elements called plasmids. Replication, vertical and horizontal transmission and evolution of these elements are discussed, and examples of the genes responsible for the resistance phenotypes are given. Selective forces that drive the evolution of new combinations of bacterial properties of particular importance in clinical situations are analysed.
\end{abstract}

\section{Introduction}

Although bacterial resistance to antibiotics must be as ancient as antibiotic synthesis, our preoccupation with it originates in very recent times and is almost exclusively focused on its impact on clinical medicine. Inherent susceptibility to antibiotics varies widely among bacteria and is often determined by the permeability properties of the cell surface, which are in most cases genetically specified by the chromosome (but see below). On the other hand, high level resistance to drugs is usually, though not always, plasmid-encoded. This is almost certainly due to the powerful selective pressures that have arisen from the widespread use of antibiotics in medicine and animal husbandry, on one hand, and to the inherent genetic plasticity of plasmids, which makes them agents par excellence of evolution and genetic dissemination, on the other. As we shall see, other types of selective pressure have ensured that many virulence determinants of pathogenic bacteria are also plasmid-carried. In this review, we shall describe briefly basic properties of plasmids, then analyse the interplay of plasmid-encoded properties that facilitate the pathogenic mode of growth of facultative pathogens, and finally consider the driving selective pressures and evolutionary trends in bacterial pathogenesis and antibiotic resistance.

\section{Basic functions of plasmids}

Plasmids are extrachromosomal genetic elements consisting of circular doublestranded DNA molecules that range in size from several to greater than 400 kilobase pairs. A high proportion of bacteria obtained from the environment or from clinical specimens carry plasmids, and many plasmid-positive isolates contain several distinct plasmid species. It is not unusual to detect eight or more different plasmids in an isolate but there seems to be an upper limit to the total amount of plasmid DNA that can be stably maintained in a host cell. Each species of plasmid is generally maintained

*This paper is dedicated to the memory of Anna Mayr-Harting. 
in exponentially-growing bacteria at a constant cellular concentration, typically between 0.7 and 40 copies per chromosome. Different plasmid species in the same bacterial cell maintain themselves at different copy numbers (Barth, Richards \& Datta, 1978), and although there is often an approximate inverse relationship between plasmid size and plasmid copy number there are many small low copy number plasmids and not a few rather large multicopy plasmids.

\section{Stable maintenance in host bacteria - vertical transfer}

Plasmids specify a wide range of bacterial activities and can freely lose and gain genetic determinants. The irreducible minimum, therefore, consists of the so-called basic replicon, which is that region of the plasmid which is essential for its stable maintenance in host bacteria, i.e. which is necessary for its perpetual vertical transmission in a growing bacterial culture. Stable maintenance necessitates plasmid determinants for initiation of plasmid replication and control of copy number (i.e. regulation of initiation of replication). It may additionally require determinants for effective distribution of plasmid copies to both daughter bacteria at cell division (partitioning), and for monomerization of plasmid multimers (Cohen et al., 1985; Nordström, 1985). Although plasmid replication depends heavily upon host chromosome DNA replication functions, regulation of replication, partitioning and monomerization seems to be largely or solely determined by plasmid genes and sequences. Independence of the regulation of plasmid and chromosome replication is exemplified by the Escherichia coli plasmid ColD, synthesis of which continues well into the stationary phase of growth, whereas that of the chromosome is tightly coupled to cell division (Frey \& Timmis, 1985). Note also that incompatibility of two related plasmids, which is a plasmid-specific property, is a consequence of cross-reacting plasmid copy number control systems (Timmis, 1979). The number of essential plasmid-specified functions for initiation of replication seems to reflect the host range of the plasmid: narrow host range plasmids like ColEl may have an origin of replication as the only essential determinant, whereas broad host range plasmids may additionally specify up to three essential functions (Scherzinger et al., 1984), reflecting the need for some degree of self-sufficiency in the initiation of plasmid synthesis in different bacteria that utilize initiation proteins having distinct specificities.

\section{Plasmid dissemination-horizontal transfer}

Although those essential maintenance determinants that constitute the basic replicon are a plasmid's only indispensable DNA sequences, most plasmids additionally carry determinants for their conjugal tranfer to new host bacteria. Conjugation itself is a complex process involving a number of different functions ( $R$. Thompson, this Volume, pp. 13-23) and is, therefore, specified only by large plasmids. Small plasmids can, however, utilize the conjugation apparatus of a co-resident conjugative plasmid if they contain an origin of transfer and specify mobilization functions which adapt the conjugation apparatus to their own transfer requirements. Although conjugation is a property of most plasmids, it is not their only means of horizontal dissemination among bacteria. Even non-conjugative, non-mobilizable plasmids can be conjugally transferred as cointegrate structures which contain a conjugative plasmid, a process that is the basis of the extensively-used gamma-delta $(\operatorname{Tn} 1000)$ mutagenesis procedure 
(Guyer, 1978). Moreover, transduction, transformation and protoplast fusion are alternative mechanisms of horizontal plasmid transfer.

Because of their propensity to transfer to (infect) new bacteria, plasmids may be considered to be intracellular parasitic or commensal organelles, and the bacteria in which they reside to be their hosts. Two types of plasmid host range should be distinguished. The strict definition relates to the range of bacteria in which a given plasmid may be stably maintained, once introduced, by whatever means. It reflects therefore the compatibility (not to be confused with plasmid incompatibility) of plasmid replication and partitioning machinery with host functions. The transfer range of a given plasmid relates to the range of bacteria to which it can be transfered by conjugation, whether or not it is subsequently inherited in the transconjugant clone. The transfer range, which is usually much wider than the host range, therefore reflects the compatibility of the plasmid conjugal transfer system with the recipient ability of the conjugal partner. Note that the disparity of host range and transfer range is the functional basis of a number of suicide transposon donor plasmids currently in use (Mermod et al., 1986).

Although plasmids and the host chromosome exhibit a high degree of physical and biochemical independence, physical interactions do occur. Indeed, integration of plasmids into host chromosomes is an important means of plasmid-mediated horizontal transfer of chromosomal genes among bacteria, as well as the incorporation, through imprecise excision, of host genes into plasmid replicons.

\section{Bacterial properties specified by plasmid determinants}

Plasmids can acquire and lose genes by integration into other replicons, followed by imprecise excision. The initial integration step may occur at a region of homology between the two replicons mediated by host recombination functions, or be mediated by an insertion element. Another means of acquiring genetic determinants is through transposition, which generally involves discrete blocks of DNA, some of which may be bracketed by copies of an insertion element. Some of these elements also exhibit high deletogenic activity and may be responsible for the loss of plasmid determinants adjacent to the point of insertion (R. Schmitt, this Volume, pp. 25-34). Plasmids collectively specify an incredibly wide range of bacterial properties ranging from conferring the ability to invade and multiply within eukaryotic cells to the degradation of herbicides. We can, however, group plasmid-encoded activities into four broad functional categories.

\section{Resistance to noxious agents}

Bacteria, like other living organisms, are continually exposed to a range of noxious agents, such as UV-irradiation and mutagenic chemicals, heavy metals, toxic organic compounds, antibiotics and lytic viruses (bacteriophages). Resistance to such agents is, however, widespread and is frequently plasmid-determined (Table I: Smith et al., 1976; Foster, 1983; Walker, 1984). It should be emphasized that resistance to antimicrobial agents utilized or produced in large quantities by man, either in medicine (e.g. antibiotics, disinfectants), agriculture (e.g. pesticides), or industry (e.g. heavy metals) tends to be widely distributed. Plasmid-mediated resistance to antibiotics is, of course, ubiquitous and the presence on single plasmids of determinants for resistance to 
Table I. Plasmid-specified bacterial resistance to noxious agents

\begin{tabular}{ll}
\hline Agent & \multicolumn{1}{c}{ Mechanism of resistance } \\
\hline Bacteriophage & $\begin{array}{c}\text { Restriction endonuclease }(E c o \text { RI }) \\
\text { degradation of infecting viral DNA } \\
\text { Modification, efflux, target } \\
\text { modification, bypass }\end{array}$ \\
Antibiotics & $\begin{array}{l}\text { Efflux, reduction, volatilization, etc. } \\
\text { Reduction, degradation, etc. }\end{array}$ \\
$\begin{array}{l}\text { Heavy metals } \\
\text { Disinfectants }\end{array}$ & $\begin{array}{l}\text { Reduced uptake } \\
\text { UV irradiation dyes } \\
\text { and chemical } \\
\text { mutagens }\end{array}$ \\
\hline
\end{tabular}

several antibiotics, as well as the location of such determinants on transposons, is well documented (e.g. Foster, 1983). Mercury compounds are used extensively in medicine as disinfectants and are also released in large quantities into the environment by industry. Almost certainly as a result of this, mercury resistance determinants are present on many plasmids, particularly antibiotic resistance plasmids, and at least one is located on a transposon (Tn501; Foster, 1983). Interestingly, resistance to acriflavine in Staphylococcus (Ericson, 1969), and to hexachlorophene in Pseudomonas (Sutton \& Jacoby, 1978), other antiseptics that have been widely used in hospitals, is also mediated by antibiotic resistance plasmids.

\section{Determinants that increase bacterial competitiveness}

Plasmids are known to specify bacterial properties whose primary function would seem to be inhibition of growth of competing micro-organisms or promotion of growth of the host organism. Bacteriocins are protein antibiotics that generally have a narrow activity spectrum and kill bacteria closely related to the producing organism, and thus, presumably, its closest competitors. Microcins on the other hand are broad spectrum oligopeptide antibiotics. Bacteriocins and microcins are generally plasmid-determined (Hopwood, 1978).

Ferric iron is a mineral essential for bacterial growth and is normally available in the environment only in minute quantities. Some bacteria secrete a siderophore, a compound having an extremely high affinity for iron, and an uptake system for the siderophore-iron complex, thereby depleting their environment of free ferric iron and effectively starving other microbes that do not produce siderophores. The aerobactintype siderophore is generally plasmid-encoded (Williams \& Carbonetti, 1985).

Adhesion antigens enable bacteria to bind specifically to certain tissues. This property considerably favours their ability to colonize such tissues over that of bacteria lacking specific adhesins, particularly where there is oriented movement of fluid across the tissue in question (e.g. mucosal surfaces), which tends to wash away looselyassociated organisms.

\section{Adaptation to special ecological niches}

Although there are many instances of plasmid-specified attributes that enable bacteria to colonize unusual ecological niches, we shall restrict discussion to those that are 
important in the clinical situation (Table II), namely the properties that enable bacteria to switch to and profit from the pathogenic mode of growth (e.g. see Timmis \& Manning, 1986). It should be emphasized that this mode involves survival in an extremely hostile environment and generally requires expression of a multiplicity of properties that provide the bacterium with resistance to a multiplicity of host defences against infection.

In order to profit from the parasitic mode, a facultative pathogen must gain access to and colonize a target tissue, resist or avoid host defences relevant to that tissue, and, ultimately, damage the host. We have already noted that cell surface adhesins which mediate adherance of bacteria to specific target cells are often plasmid-specified. The K88, K99 and CFA antigens of porcine, bovine and human enterotoxigenic strains of $E$. coli permit bacteria which specify them to adhere to and colonize the small bowel epithelium.

Table II. Plasmid-specified adaptation to particular ecological niches

\begin{tabular}{ll}
\hline Plasmid function & Ecological niche \\
\hline Antibiotic resistance & Hospitals \\
Disinfectant resistance & Hospitals \\
Adhesin of ETEC & Ileal epithelium \\
Aerobactin siderophore & Body fluids containing \\
& transferrin, lactoferrin \\
"Invasion factors" of & Cytoplasm of invaded \\
Shigella spp. and EIEC & epithelial cells \\
Complement resistance factors & Blood, surfaces bathed \\
(traT protein; iss protein; & by complement-containing \\
O-antigen of Shigella) & fluids \\
Phagocytosis resistance factors & Blood, phagocyte- \\
V \& W antigen of Yersinia; & accessible tissues \\
capsule of $B$. anthracis & \\
\hline
\end{tabular}

ETEC, enterotoxigenic $E$. coli; EIEC, enteroinvasive $E$. coli.

Enterotoxigenic bacteria damage the host by releasing enterotoxins which act on the epithelium and cause fluid secretion (diarrhoea). There are two families of enterotoxins of Gram-negative enteric bacteria: small molecular weight heat-stable polypeptide enterotoxins (ST), some of which activate guanylate cyclase in epithelial cells, and larger molecular weight heat-labile protein enterotoxins (LT and CT [cholera toxin]), which activate adenylate cyclase. Enterotoxins are largely, though not exclusively, plasmid-encoded in $E$. coli and several enterotoxin determinants are located on transposable elements (e.g. Tn1681).

If many environments have low levels of free iron, body fluids have essentially none as a result of their containing large amounts of iron-binding proteins; transferrin in blood and lactoferrin in secretions. Colonization of mucosal surfaces or internal tissues is, therefore, not possible for many bacteria. The siderophores of pathogenic bacteria, however, are able to capture iron from the body's iron binding proteins and are, as a consequence, important virulence factors (Williams \& Carbonetti, 1985). As indicated above, aerobactin production and transport is plasmid-encoded in some strains. Acquisition of the aerobactin-multiple antibiotic resistance plasmid FIme by 
Salmonella spp. has been proposed as the reason for the emergence of epidemic strains exhibiting enhanced virulence (Colonna et al., 1985).

Whereas enterotoxigenic bacteria generally cause disease by intoxication from without, other bacteria invade the body. Shigellae and enteroinvasive E. coli, for example, penetrate and multiply within large bowel epithelial cells, ultimately killing them and causing mucosal ulceration. The ability to invade and multiply in epithelial cells is always specified by a large plasmid that is readily lost during laboratory cultivation of these enteroinvasive bacteria (Sansonetti et al., 1986; Timmis, Sturm \& Watanabe, 1986).

Once a pathogen has caused damage, inflammatory factors are induced and the invading organism is confronted with a battery of host defences that include complement and phagocytes. Prolongation of the infection, i.e. the parasitic mode, requires bacterial resistance to these defences. Resistance to complement and phagocytosis may be mediated by O-specific polysaccharide of LPS, acidic polysaccharide capsules, and outer membrane proteins of Gram-negative bacteria (Timmis et al., 1985), and by polypeptide capsules and fibrilar surface structures (Mproteins) of Gram-positive bacteria. The O-antigens of two shigellae are partly or entirely plasmid-determined (Timmis et al., 1986), as are the $\mathrm{V}$ and $\mathrm{W}$ antigens of Yersinia (Brubaker, 1985), the traT and iss outer membrane proteins of E. coli and its relatives (Timmis et al., 1985), and the capsule of Bacillus anthracis (Uchida et al., 1985). Thus, plasmid-determined factors can play key roles at various stages in infections.

It is interesting that expression of some resistance factors is activated by host parameters (Sansonetti et al., 1986; Timmis \& Manning, 1986). Body temperature, for instance, induces the synthesis of Shigella proteins necessary for invasion of and multiplication within epithelial cells, Yersinia proteins involved in resistance to host defences, and $E$. coli colonization antigens; (none of these are synthesized in significant amounts at $30^{\circ} \mathrm{C}$ or below). Even more remarkable is the finding that a major host defence, iron deprivation, induces expression of some virulence factors, including siderophore production and the synthesis of some cytotoxins and cytolysins.

\section{Evolution of new properties and new combinations of properties}

Plasmid-mediated acquisition of bacterial resistance to new combinations of antibiotics, and the rapid dissemination of these combinations to new bacteria are classical examples of plasmid-mediated evolution of bacterial properties. Antibiotic resistance probably has several different origins, but some resistances are almost certainly derived from antibiotic-producing organisms which, of necessity, must protect themselves from the action of the antibiotics they synthesize. Comparison of the amino acid sequences of aminoglycoside- 3 '-phosphotransferases that modify kanamycin and neomycin and that are synthesized by neomycin-producing Streptomyces fradiae, aminoglycoside-resistant Staphylococcus aureus and E. coli strains containing transposon $\operatorname{Tn} 5$ or $\operatorname{Tn} 903$ has revealed considerable homology between the different enzymes and suggests that the ancestral Strep. fradiae protein may be the origin of this class of antibiotic inactivating enzymes (Foster, 1983; Davies \& Gray, 1984).

One plausible route for the development of some drug resistance determinants is shown in Figure 1. Within the microenvironment in which an antibiotic-producing 


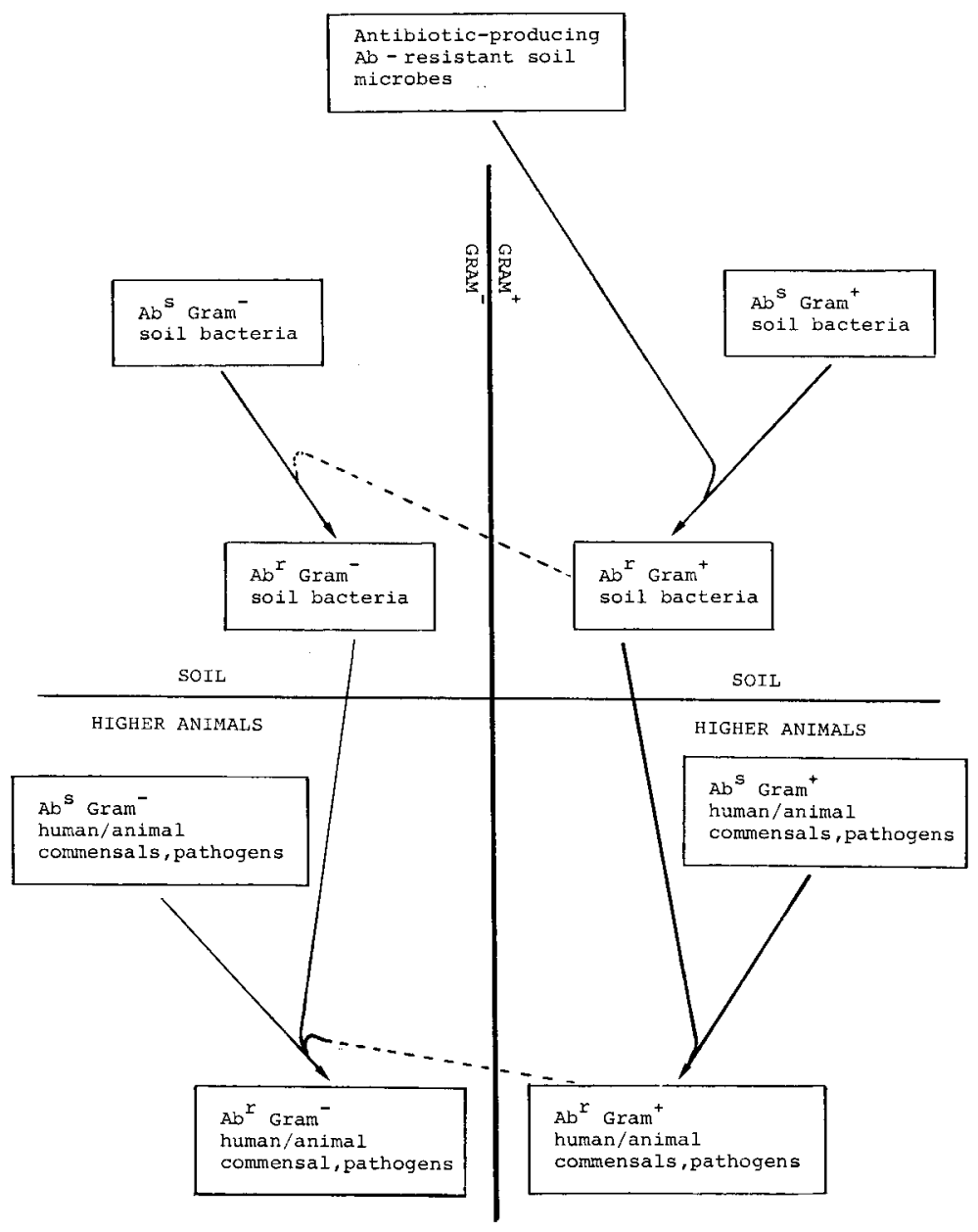

Figure 1. Possible evolutionary route of resistance to some antibiotics in bacterial pathogens.

organism grows, there is strong selective pressure for other micro-organisms to develop resistance to the antibiotic; this may be acquired through transfer of resistance genes from the producer to appropriate recipients, for example Gram-positive bacteria. These would, in turn, transfer the resistance genes to Gram-positive human or animal commensals and pathogens. Presumably at very low frequency, there is also transfer to Gram-negative bacteria, perhaps by transformation or protoplast fusion. This may occur among soil bacteria or among commensals, or both. The recent finding of a typical Gram-positive gentamicin-inactivating enzyme in Campylobacter (Trieu-Cout et al., 1985) supports the notion that the genetic exchange barrier between Grampositive and Gram-negative bacteria, though formidable, is not absolute.

$\beta$-Lactamases may have evolved by a different route (Tipper \& Strominger, 1965). $\beta$-Lactam antibiotics kill bacteria by inhibiting a set of penicillin-binding proteins (PBPs) which are serine enzymes that are bound to the outer face of the cytoplasmic membrane and that catalyse the final stages of peptidoglycan synthesis (Spratt, 1983; Waxman \& Strominger, 1983). Many PBPs having D-alanine carboxypeptidase activity 
exhibit low $\beta$-lactamase activities. Comparison of the amino acid sequences of class $\mathrm{A}$ $\beta$-lactamases, which are also serine enzymes and which include the TEM $\beta$-lactamase, and PBPs 5 and 6 of E. coli and PBP5s of Bacillus subtilis and B. stearothermophilus has revealed a high degree of homology in the region of the active site serine (Spratt, 1983). The $B$. subtilis and B. stearothermophilus PBP5 carboxypeptidases are known to be ectoproteins which can be cleaved from the cell membrane with trypsin to form water-soluble enzymes that have lost a small $\mathrm{COOH}$-terminal hydrophobic membrane anchoring peptide (Waxman \& Strominger, 1979, 1983). Ancestral carboxypeptidases might therefore have evolved into current class A $\beta$-lactamases through loss of the anchor peptide and by augmentation of hydrolytic activity towards $\beta$-lactams.

Whatever the origin of individual resistances, it is clear that their widespread dissemination, particularly into pathogenic bacteria, and the evolution of strains carrying multiple resistances are recent phenomena (Datta, 1985) and directly result from the pervasive, often indiscriminate use of antibiotics. Similarly, mercury resistance was a rare plasmid-specified property prior to the $1960 \mathrm{~s}$, although it is an extremely prevalent property of current plasmids, particularly antibiotic resistance plasmids (Foster, 1983). By effecting horizontal transfers and recombinational events, plasmids have played a key role in the evolution of resistance combinations on single replicons.

Enhancement of virulence properties also occurs under appropriate selection pressure. A well-known phenomenon is the reduction in levels of enterotoxin produced by enterotoxigenic bacteria during passage in the laboratory, and the increase in levels during passage through an experimental animal. This was recently shown in Vibrio cholerae to result from deletion or amplification of cholera toxin genes, mediated by insertion sequences flanking these genes, and the resulting gene dosage effect (Mekalanos, 1985). Although cholera toxin is specified by chromosomal determinants, many other enterotoxins are plasmid-encoded and we can assume that similar mechanisms operate in the evolution of enhanced toxin synthesis under conditions that result in high frequencies of transmission of the infectious agents. The increasing occurrence of plasmids containing multiple determinants of distinct enterotoxins $($ LT + ST; STa + STb; Table III; Gyles, Palchaudhuri \& Maas, 1977; Echeverria et al., 1985 ) is also consistent with a tendency towards evolution of enhanced virulence.

\section{Conclusions and outlook}

Plasmids are non-essential genetic elements that can on occasion provide host bacteria with enormous selective advantage. They therefore increase bacterial adaptability in the short term. Because of their capacity to transfer horizontally among bacterial populations (epidemically under appropriate selective conditions), they are the principal means of gene transfer in most bacteria and, because they frequently contain insertion elements and transposons, they constitute a major mechanism of gene reassortment. Plasmids are thus principal agents of evolution and of bacterial adaptability in the long term.

Although many different highly selective environmental conditions exist that have been created by man and modern living (e.g. the release of enormous quantities of xenobiotic compounds as pesticides), we only consider three which relate to the evolution of clinically important bacteria. Firstly, widespread use of antibiotics has resulted in the evolution of conjugative multi-drug resistance plasmids, and these are 
increasingly compromising effective treatment of a variety of infections (I. Philips, this Volume, pp. 255-60). Secondly, current socio-economic situations result in conditions of overcrowding and poor hygiene for certain groups of individuals, such as some communities in developing countries, infants attending daycare centres, and intensively-reared farm animals. Such conditions result in extremely high frequencies of transmission of infectious diseases within these groups. Moreover, infections in developing countries and of intensively-reared farm animals are often exacerbated by poor resistance levels to infection. Situations of this type favour the pathogenic mode of growth of facultative parasites and constitute a powerful selective force for the evolution of enhanced virulence and of plasmids containing multiple virulence determinants. Finally, hospitals constitute unique selective environments because a multitude of antimicrobial drugs and antiseptics are in daily use, and because they are the institutions in which new drugs are first employed. Hospitals therefore select not only for the evolution of plasmids containing multiple determinants of resistance to drugs and antiseptics, but also for the evolution of resistance to new drugs. Collectively, these and other selective forces favour the evolution of individual plasmids carrying determinants for resistance to multiple drugs and antiseptics, and for multiple virulence factors (Table III; Gyles et al., 1977; Mullany et al., 1983; Echeverria et al., 1985; Manning, Timmis \& Stevenson, 1985), because with such plasmids fewer genetic events are required to transfer enhanced pathogenic potential to new bacteria, particularly within a hospital environment. The location on transposons or transposon-like structures of many determinants, for example those of certain antibiotic resistances, enterotoxins, adhesion antigens, iron uptake systems, and heavy metal resistances, presumably facilitates this process. It is self-evident that because of the formation of antibiotic resistance plasmids carrying virulence determinants, antibiotic usage (both clinically and non-clinically) will select and favour dissemination of virulence functions among bacteria and thus facilitate the evolution of strains with enhanced virulence.

Table III. Multiplicity of determinants of virulence and of drug and antiseptic resistance on individual plasmids of intestinal pathogens

\begin{tabular}{lll}
\hline Bacterium & Source & \multicolumn{1}{c}{ Plasmid phenotypes } \\
\hline E. coli & porcine & $\mathrm{STb}, \mathrm{LT}, \mathrm{Tc}^{\mathrm{r}}, \mathrm{Sm}^{\mathrm{r}}, \mathrm{Su}^{\mathrm{r}}, \mathrm{Hg}^{\mathrm{r}}$ \\
& human & $\mathrm{ST}, \mathrm{CFA} / \mathrm{I}$ \\
& human & $\mathrm{ST}, \mathrm{CFA} / \mathrm{II}$ \\
& human & $\mathrm{STb}, \mathrm{Ap}, \mathrm{Cm}^{\mathrm{r}}, \mathrm{Tc}^{\mathrm{r}}, \mathrm{Sm}^{\mathrm{r}}, \mathrm{Su}^{\mathrm{r}}$ \\
& human & $\mathrm{STa}, \mathrm{STb}$ \\
& human & $\mathrm{STb}, \mathrm{LT}, \mathrm{Tc}^{\mathrm{r}}$ \\
Salmonella wien & human & $\mathrm{ST}, \mathrm{K} 99, \mathrm{Hc}^{\mathrm{r}}, \mathrm{Sm}^{\mathrm{r}}, \mathrm{Su}^{\mathrm{r}}$ \\
Shigella sonnei & human & Invasion, $\mathrm{C} \mathrm{Cantigen}^{\mathrm{r}}, \mathrm{Gm}^{\mathrm{r}}, \mathrm{Hg}^{\mathrm{r}}$ \\
Shig. flexneri & human & Invasion, $\mathrm{Hly}$ \\
\hline
\end{tabular}

ST, LT, Heat stable and heat labile enterotoxins, respectively; $\mathrm{K}$ and CFA, colonization (adhesion) antigens; $\mathrm{Ap}^{\mathrm{r}}, \mathrm{Cm}^{\mathrm{r}}, \mathrm{Gm}^{\mathrm{r}}, \mathrm{Km}^{\mathrm{r}}, \mathrm{Tc}^{\mathrm{r}}$, $\mathrm{Sm}^{\mathrm{r}}, \mathrm{Su}^{\mathrm{r}}, \mathrm{Hg}^{\mathrm{r}}$, resistance to ampicillin, chloramphenicol, gentamycin, kanamycin, tetracycline, streptomycin, sulphonamide, and mercury, respectively; Hly, haemolysin; Hds, hydroxamate synthesis (aerobactin). 
From the foregoing it is clear that plasmids of clinically-important bacteria are important vectors of bacterial aggressions and of multivalent resistance to intrinsic and extrinsic human defences to infection. Although this multivalence usually reflects the activity of multiple cellular products, the traT protein specified by IncF group conjugative antibiotic resistance plasmids is a single polypeptide that exhibits multifunctionality (Timmis et al., 1985). The traT protein is located on the outer surface of the outer membrane of $E$. coli and mediates (a) surface exclusion, a plasmid conjugal transfer function, (b) bacterial resistance to complement, a virulence-related function, and (c) resistance to hydrophobic antibiotics such as erythromycin, which can be considered to be a hospital environment-related function (Sukupolvi, S., O'Connor, D. \& Edwards, W. F., unpublished results). Functional analysis of traT and epitope mapping, using gene fusions and monoclonal antibodies directed against non-cell surface-located epitopes, has indicated that the three functions are mediated by distinct domains of the protein and that these are distinct from the three epitopes recognized by the monoclonal antibodies, and which are assumed to be located in transmembrane domains (O'Connor, C. D., Bitter-Suermann, D. \& Timmis K. N., in preparation). Thus, the traT protein may be an example of the evolution of a single gene product of an antibiotic resistance plasmid towards multiple functions that are pertinent to a bacterial pathogen.

\section{Acknowledgements}

It is a pleasure to acknowledge Julian Davies for informative discussion and Françoise Rey for valued secretarial assistance. Work described here that was carried out in the author's laboratory was supported by grants from the Swiss National Science Foundation and the Diarrhoeal Diseases Control Programme of the World Health Organization.

\section{References}

Barth, P. T., Richards, H. \& Datta, N. (1978). Copy numbers of coexisting plasmids in Escherichia coli K-12. Journal of Bacteriology 135, 760-5.

Brubaker, R. R. (1985). Mechanisms of bacterial virulence. Annual Reviews of Microbiology 39, $21-50$.

Cohen, S. N., Miller, C. A., Tucker, W. T., Meacock, P. A. \& Gustafsson, P. (1985) Partitioning of the pSC101 plasmid during cell division. In Plasmids in Bacteria (Helinski, D. R., Cohen, S. N., Clewell, D. B., Jackson, D. A. \& Hollaender, A., Eds), pp. 383-95. Plenum Press, New York.

Colonna, B., Nicoletti, M., Visca, P., Casalino, M., Valenti, P. \& Maimone, F. (1985). Composite IS 1 elements encoding hydroxymate-mediated iron uptake in FIme plasmids from epidemic Salmonella spp. Journal of Bacteriology 162, 307-16.

Datta, N. (1985). Plasmids as organisms. In Plasmids in Bacteria (Helinski, D. R., Cohen, S. N., Clewell, D. B., Jackson, D. A. \& Hollaender, A., Eds), pp. 3-16. Plenum Press, New York.

Davies, J. \& Gray, G. (1984). Evolutionary relationships among genes for antibiotic resistance. In Origins and Development of Adaptation, Ciba Foundation Symposium 102 (Evered, D. \& Collins, G. M., Eds), pp. 219-28. Pitman, London.

Echeverria, P., Sekiwatana, J., Taylor, D. N., Tivapat, C. \& Rowe, B. (1985). Escherichia coli contains plasmids coding for heat-stable $b$, other enterotoxins and antibiotic resistance. Infection and Immunity 48, 843-6.

Ericson, C. (1969). Resistance to acriflavine and cadmium, and changed phase reactionsmarkers of a new staphylococcal penicillinase plasmid. Acta Pathologica et Microbiologica Scandinavica 76, 333 . 
Foster, T. J. (1983). Plasmid-determined resistance to antimicrobial drugs and toxic metal ions in bacteria. Microbiological Reviews 43, 361-409.

Frey, J. \& Timmis, K. N. (1985). ColD-derived cloning vectors that autoamplify in the stationary phase of bacterial growth. Gene 35, 103-11.

Guyer, M. (1978). The $\gamma \delta$ sequence of $F$ is an insertion sequence. Journal of Molecular Biology 126, 347-65.

Gyles, C. L., Palchaudhuri, S. \& Maas, W. K. (1977). Naturally occurring plasmid genes for enterotoxin production and drug resistance. Science 98, 198-9.

Hopwood, D. A. (1978). Extrachromosomally-determined antibiotic production. Annual Review of Microbiology 32, 373-92.

Manning, P. A., Timmis, K. N. \& Stevenson, G. (1985). Colonization factor antigen II (CFA/II) of enterotoxigenic Escherichia coli: molecular cloning of the CS3 determinant. Molecular and General Genetics 200, 322-7.

Mekalanos, J. J. (1985). Cholera toxin: genetic analysis, regulation and role in pathogenesis. Current Topics in Microbiology and Immunology 118, 97-118.

Mermod, N., Lehrbach, P. R., Don, R. H. \& Timmis, K. K. (1986). Gene cloning and manipulation in Pseudomonas. In The Bacteria, Vol. 10, The Biology of Pseudomonas.

Mullany, P., Field, A. M., McConnell, M. M., Scotland, S. M., Smith H. W. \& Rowe, B. (1983). Expression of plasmids coding for colonization factor antigen II (CFA/II) and enterotoxin production in Escherichia coli. Journal of General Microbiology 129, 3591-601.

Nordström, K. (1985). Replication, incompatibility and partition. In Plasmids in Bacteria (Helinski, D. R., Cohen, S. N., Clewell, D. B., Jackson, D. A. \& Hollaender, A., Eds), pp. 119-123. Plenum Press, New York.

Sansonetti, P. J., Ryter, A., Clerc, P., Maurelli, A. \& Mounier, J. (1986). Multiplication of Shigella flexneri within HeLa cells: Lysis of the phagocytic vacuole and plasmid-mediated contact hemolysis. Infection and Immunity 51, 461-9.

Scherzinger, E., Bagdasarian, M. M., Scholz, P., Lurz, R., Rückert, B. \& Bagdasarian, M. (1984). Replication of the broad host range plasmid RSF1010: requirement for three plasmid-encoded proteins. Proceedings of the National Academy of Sciences U.S.A. 81, $654-8$.

Smith, H. R., Humphreys, G. O., Willshaw, G. A. \& Anderson, E. S. (1976) . Characterization of plasmids coding for the restriction-endonuclease EcoRI. Molecular and General Genetics $143,319-25$.

Spratt, B. G. (1983). Penicillin binding proteins and the future of $\beta$-lactam antibiotics. Journal of General Microbiology 129, 1247-60.

Sutton, L. \& Jacoby, G. A. (1978). Plasmid-determined resistance to hexachlorophene in Pseudomonas aeruginosa. Antimicrobial Agents and Chemotherapy 13, 634-6.

Timmis, K. N. (1979). Mechanisms of plasmid incompatibility. In Plasmids of Medical, Environmental and Commercial Importance (Timmis, K. N. \& Puhler, A., Eds), pp. 13-21. Elsevier/North-Holland Biomedical Press.

Timmis, K. N., Boulnois, G. J., Bitter-Suermann, D. \& Cabello, F. C. (1985). Surface components of Escherichia coli that mediate resistance to the bactericidal activities of serum and phagocytes. Current Topics in Microbiology and Immunology 118, 197-218.

Timmis, K. N. \& Manning, P. A. (1986). Virulence factors of enteropathogenic bacteria. In Bacterial Protein Toxins, in press.

Timmis, K. N., Sturm, S. \& Watanabe, H. (1986). Genetic dissection of pathogenesis determinants of Shigella and enteroinvasive Escherichia coli. In Development of Vaccines and Drugs against Diarrhea (1 Ith Nobel Conference, Stockholm 1985) (Holmgren, J., Lindberg, A. \& Mollby, R., Eds), pp. 107-26. Studentlitteratur, Lund, Sweden.

Tipper, D. J. \& Strominger, J. L. (1965). Mechanism of action of penicillins: a proposal based on their structural similarity to acyl-D-alanyl-D-alanine. Proceedings of the National Academy of Sciences U.S.A. 54, 1133-41.

Trieu-Cuot, P., Gerbaud, G., Lambert, T. \& Courvalin, P. (1985). In vivo transfer of genetic information between Gram-positive and Gram-negative bacteria. EMBO Journal 4, 3583-7.

Uchida, I., Sekizaki, T., Hashimoto, K. \& Terakado, N. (1985). Association of the encapsulation of Bacillus anthracis with 60 megadalton plasmid. Journal of General Microbiology 113, 363-7. 
Walker, G. C. (1984). Mutagenesis and inducible responses to deoxyribonucleic acid damage in Escherichia coli. Microbiological Reviews 48, 60-93.

Waxman, D. J. \& Strominger, J. L. (1979). Cleavage of a COOH-terminal hydrophobic region from D-alanine carboxypeptidase, a penicillin sensitive bacterial membrane enzyme. Journal of Biological Chemistry 254, 4863-75.

Waxman, D. J. \& Strominger, J. L. (1983). Penicillin binding proteins and the mechanism of action of $\beta$-lactam antibiotics. Annual Review of Biochemistry 52, 825-69.

Williams, P. H. \& Carbonetti, N. H. (1985). The plasmid-specified aerobactin iron uptake system of Escherichia coli. In Plasmids in Bacteria (Helinski, D. R., Cohen, S. N., Clewell, D. B., Jackson, D. A. \& Hollaender, A., Eds), pp. 741-57. Plenum Press, New York. 